\title{
Analysis of Equivalent Structures in Persian for the Translation of English Passive Sentences Based on Translations of "Animal Farms"
}

\author{
Omid Tabatabaei \\ English Department, Najafabad Branch, Islamic Azad University, Najafabad, Iran \\ Email: tabatabaeiomid@yahoo.com \\ Ashraf Rostampour \\ Persian Literature Department, Najafabad Branch, Islamic Azad University, Najafabad, Iran
}

\begin{abstract}
This article is an attempt to analyze the Persian language structures equivalent to the Passive structures in English. After a brief review of both linguists and grammarians views about this structure, various structures used in the translations of the English passive structure in the book "Animal Farm" by George Orwell (1945) have been analyzed resulting in the introduction eleven different categories of such structures. Then the frequency of each structure has been calculated in each of the five available translations according to which the more frequent structures were introduced. The findings show that the structure "active-intransitive verbs" is the most common structure in these translations which is used to translate the passive structure in English. The findings also confirm the previous research claiming that in the Persian language there is a tendency to use the active structure rather than the passive one.
\end{abstract}

Index Terms—passive, Persian, English, equivalent structures, style

\section{INTRODUCTION}

Speakers of any language use all the means available in their language to convey their meaning. Because of the variety of such means in different languages, when translating a text from one language to another, it may not be possible to use the same capacities available in the source language. One of the structures which have been a cause of dispute among different grammarians and linguistics is the "passive voice". It is of more challenge when translating a text from the English language, whose speakers tend to use this structure more often, to another one. This difference in the frequency of this structure between the two languages, namely English and Persian, can be one of the basic differences between the two languages. This difference becomes more conspicuous when translating from one language into another. The failure to pay attention to these differences has led to the translation of passive voice in English to a passive voice in Persian, which makes the translated texts seem odd and unusual in Persian.

On the other hand, there are different ideas about this structure in Persian. Considering the form and structure, there is a "past participle of the verb +a form of the verb "become" [shodan] which all researchers in this field believe to be the equivalent for the passive structure in English. In this article different available structures in Persian used to translate the passive structure in English are analyzed and their frequencies are identified according to different translations of the book "Animal Farm" by Orwell (1945). These translations include:

Translation 1: Huria Moosaei (2009), Translation 2: Alizade Z.(2008), Translation 3: Amir Amirshahi (2009), Translation 4: Muhammad Firoozbakht (2009), Translation 5: Saleh Hosseini \& Masumeh Nabizadeh (2008)

\section{LITERATURE REVIEW}

Passive voice, like many other aspects of language first appeared in Greek grammarians' works. They were the first who developed the idea of aspect (derived from vox meaning form) and by combining this idea with active and passive, they discussed the different forms of active and passive sentences (Lyons, 1996). It is worth paying attention to the Greek tradition because of their deep influence on the succeeding grammarians including the Romans as well as the contemporary western and non-westerns. Their focus on the form of passive structure and the idea that this structure is derived from the active one led to their inability to comprehensively account for the Greek grammar, and also caused their succeeding grammarians to consider the passive structure as the one derived from the active one and not to think of anything beyond it. With this in mind, passive and its structure has been of great importance to researchers. Different studies show that the frequency of occurrence of this structure in English and some other European languages is more than that in Persian (Jabbari, 2003). Linguists and grammarians have different viewpoints about this structure; grammarians consider a fixed and certain form as passive and they only consider form to distinguish active from passive but linguists think of other structures in addition to the form of passive structure. Those structures are the ones 
that have the meaning of passive structures but their form is not passive; therefore, for them the meaning of a structure is as important as its form. As a result their viewpoints can be categorized as follows:

\section{A. Passive in Persian}

\section{Form and Structure}

Passive and its structure is one of the issues grammarians pay attention to and they have a special definition for passive and its structure. "A passive verb is attributed to the direct object like: Hassan was seen. [Hassan dide shod] in which "was seen" [dide shod] is passive and is attributed to the direct object, Hassan. (Shariat, 1988, p.165). Khanlari (1998) believes that the difference between active and passive lies in the attribution of the verb to the subject and object, respectively. He also believes that the theme in an active sentence is the subject and in a passive one is the object. (pp. 84-85)

Anvari \& Givi (1996) define passive as a verb whose subject is not known. In other words, we can attribute a transitive verb to the object without mentioning its subject. For example in the sentence: The teacher was seen in the street. [Moallem dar khiaban dide shod] the verb "was seen" [dide shod] is attributed to the object; in other words the theme of the sentence is the object rather than the subject. As it is only the transitive verb that can have an object, the passive verb is always derived from a transitive verb. The passive verb consists of the past participle (past base form of the verb + the final vowel sound/e/) and one form of the auxiliary verb "become" [shodan] (p.50). They believe that verbs like "be released" [raha shodan], "be found" [peida shodan], and "be lost" [gom shodan] are the intransitive forms of the transitive verbs "release" [raha kardan], "find" [peida kardan], and "lose" [gom kardan], respectively (p.26).

Lambton (1983) believes that in Persian, if it is possible to use the active structure, the passive one will not be used. So the sentence "I was hit by him." should be translated as "He hit me." [ou mara zad] (p.54). Some others deny the existence of a passive structure in Persian. In John Moyne's idea (1974) the distinction between active and passive structures does not exist in Persian but there are some intransitive verbs accompanied by the verb "become" [shodan]. Some other researchers in this field limit the application of passive structures to cases where there is no agent (doer of the action). In Soheili's idea (1976) through the process of passivization the direct object is extraposed to the place of the subject and the subject is generally omitted.

In his article "Passive in Persian" Dabirmoghaddam (1985) makes a syntactic distinction between the structure of passive and intransitive verbs and states that the passive transformation is a limited one applied just to verbs whose subject has control over the verb. The application of the passive transformation to the deep structure of active sentences is comparable with the three transformations changing the active to passive sentences. In modern Persian, the intransitive motion verb of "go" [shodan] has turned into an auxiliary verb used in passive sentences. On the other hand, a group of causative sentences made up of an adjective and the causative verb "do" [kardan] has also something to do with the auxiliary verb "become" [shodan].

Lazar (2005) states that a passive verb is made by using the past participle of the verb and the auxiliary verb "become" [shodan]. (pp. 191-192)

Vahidian Kamyar (2005) argues that what all grammarians believe to be the past participle of the verb is derived by adding a final vowel /e/ to the past base form of the verb. But most of these so-called "past participles" of the verbs are neither an adjective nor even a free morpheme. For example, grammarians consider the form "beaten" [zade] as the past participle of the verb "beat" [zadan], while [zade] is neither an adjective nor even a free morpheme and it cannot replace another adjective. For example, it is not possible to have the phrase "a beaten child" [bacheye zade] or to replace it for a noun like "the beaten left" [zadeha raftand]. The past participle of the verb "wash" [shostan], however, is "washed" [shoste] and can be used in the phrase "washed clothes" [lebashaye shoste]. He believes that in Persian the morpheme $[a n d]$ and the active form of the verb rather than using the passive structure is used if the doer of the action is unknown.

\section{Viewpoints on Meaning and Function}

The word "function" in this group of works is used to be in contrast with "structure". Bateni (1969) considers other structures in addition to the form of passive (past participle and the auxiliary "become" [shodan]) to state the passive meaning. He believes there are three other ways to express a passive meaning:

1. The non-personal aspect, like "He should be seen." [ou ra bayad did]

2. The third person plural form of the verb without mentioning the subject, like "They say he is a good person." [miguyand adame khubi ast], or "they beat him" [zadandash]

3. Changing the verb of the sentence so that the structure of the sentence will not change but the verb will. For example, the verb "do" [kardan] will change to "become" [shodan] and "beat" [zadan] to "eat" [khordan], like "He announced the result" [natije ra elam kard] to "the result was announced" [natije elam shod] (p. 136)

Pakravan (2002) puts the word "function" as opposed to "structure" and believes that we should first clarify the function of passive and then distinguish it from other structures in Persian. The function of passive is the one in which the agent or the doer of the action is of the secondary importance compared with the direct object, purpose or the action itself. The way of decreasing the importance of the agent is different from one language to another. In Persian because the sequence of the words in a sentence is not so strict, and also because of the possibility of subject omission, the bound morpheme in the verb standing for the subject and the morpheme $[\mathrm{ra}$ ] which shows the direct object in a 
sentence, the motivation for the application of the passive structure is different and it is used not to decrease the level of the subject but to omit it. In cases where the purpose is to decrease the level of importance of the subject, it is possible to start the sentence with the direct object and mention the subject after that. For example, it is possible to have the sentences "Hassan stole the car." [mashin ra Hassan dozdid] or "He stole the car." [mashin ra dozdid]. It is also possible to add the prepositional phrase "by ..." [tavassote ...], like "the car was stolen by Hassan." [ mashin tavassote Hassan dozdide shod]

\section{B. Passive in English}

Quirk (1985) states that in the active voice, the subject of the sentence performs the action of the sentence. An active sentence is subject + verb + object. Take this sentence for example: The boy hit the ball. "Boy" is the subject of the sentence, and "hit" is the verb. Since the subject of the sentence, boy, is doing the action, hitting the ball, this sentence is said to be in the active voice. The passive voice, on the other hand, is the opposite. In a passive sentence, the subject of the sentence receives the action of the verb, like: The ball was hit by the boy. In this sentence, the subject, ball, is not performing the action of the sentence. The ball is not the agent doing the hitting. Instead, it is receiving the action of the sentence. The ball is the subject receiving the action of the verb.

To simplify, the passive is formed when the active subject becomes the passive agent; the active object becomes the passive subject; and the preposition "by" is introduced before the agent (pp. 159-165).

\section{METHOdOLOGY}

As the purpose of this study was to analyze the available means in Persian to translate English passive sentences and their frequencies, we needed some practical and suitable data. It seems that novels and short stories are more appropriate because they include various adventures and dialogs. The story “Animal Farm” by George Orwell (1945) was chosen as a source to identify and analyze passive structure. This book has different translations in Persian which are suitable for our aim to deal with different structures used in Persian to translate those sentences. Five translations were chosen and then the passive sentences were selected in the ten chapters of the original book, afterwards their equivalent structures in the five translations were indentified and categorized. These structures were categorized according to their form and the definitions put forward by Anvari \& Givi(1996) and then they were put in different eleven groups of structures which would be discussed in the following section.

\section{ANALYZING THE STRUCTURES}

The following groups of structures and their examples, extracted from the data in the original book and the translations, would make the point of focus in this study clear:

\section{A. Group One: Active Structure}

In this group, the English passive structures, which are translated through active sentences in Persian, are discussed:

Intransitive Verbs:

According to Anvari \& Givi (1996, p.64), a verb which completes the meaning of a sentence without an object or does not need an object is an intransitive one like "went" [ raft], "stood up" [barkhast], and "sat down" [neshast]

The researchers in this study have divided the intransitive verbs to two groups of linking verbs and main verbs when translating the passive verbs in English:

\section{Linking verbs:}

Linking verbs in Persian, according to Anvati and Givi, p.68), are defined as "those which do not have a perfect meaning and are just used to prove or disprove a relationship and their meaning is complete with an adjective or another word, like "It is bright." [hava roshan ast]. To clarify this point let's consider one of the examples in "Animal Farm":

1) The animals were satisfied that they had been mistaken. (Orwell, 1945, p 58)

In this sentence the passive verb "were satisfied" is translated as "were happy and pleased" [khoshhal va razi budand] in Moosaei's translation (2009, p.20)

\section{Main Verbs:}

The second group in intransitive verbs are named as intransitive-main verbs. Anvari and Givi (1996, p.64) have defined such verbs as "Most of the verbs used in a language like "go, sit, sleep" [raftan, neshastan, khabidan] show an action being done or receiving a certain action. Such verbs are called main verbs. The intransitive-main verbs are subdivided to two subcategories:

\section{Non-personal intransitive main verbs:}

Anvari and Givi (p.70) state that quasi auxiliaries like "can, must and should" [tavanestan, beyestan, and shayestan] sometimes make a verb which is not attributed to a certain person, like "can't be left, mustn't be said, shouldn't be left" [natavan raft, nabayad goft, and nashayad raft]. Such verbs are called non-personal verbs. As an example in "Animal Farm" look at the following sentence:

2). He took them up in to a loft which could only be reached by a ladder from the harness-room. (Orwell, 1945, p32)

In this sentence the passive verb "could only be reached" is translated as "was possible to go there" [mishod be anja raft] in Firuzbakht's translation (2009, p.88) 


\section{Personal Intransitive-main Verbs:}

Based on the definition offered for intransitive verbs and also the division of this group to personal and non-personal verbs, all intransitive verbs apart from the non-personal ones are categorized in the group of personal intransitive verbs. The following is an example:

3) The plan which Snowball had drawn on the floor of the incubator shed had actually been stolen from among Napoleon's papers. (Orwell, 1945, p 52)

In this sentence the passive verb "had been stolen" is translated as "had gone stolen" [be serghat rafte bood] in Alizade's translation (2008, p.87)

When analyzing the Persian translations for passive sentences, we came across some structures which are, according to Anvar \& Givi (1996), compound verbs whose second part is "become" [shodan], like "get lost" [gom shadan], "be found" [peida shodan], and "be forbidden" [man' shodan] and are believed to be the intransitive form of the compound verbs whose second part is "do" [kardan], like "lose" [gom kardan], "find" [peida kardan], and "forbid" [man' karadan]. (p. 26) Therefore, such structures which are used by translators to translate passive sentences are categorized in the group of personal intransitive verbs, like:

4).The pigeons who were still sent out to spread tidings of the Rebellion were forbidden to set foot anywhere on Foxwood. (Orwell, 1945, p 97)

In this sentence the passive structure "were forbidden" is translated as "became forbidden" [ man' shodand] in Alizade's translation (p.90).

\section{Transitive Verbs:}

The second group of active verbs includes transitive verbs. According to Anvari \& Givi (1966, p.64) a verb whose meaning is not complete without an object and needs an object to have a complete meaning and passes the subject to get to the object is called a transitive verb. In order to include more details, this group is subcategorized to two minorcategories:

\section{Non-personal Transitive verbs:}

As it was mentioned before, the quasi auxiliaries "can" [tavanestan], "must" [bayestan], and "should" [shayestan] are sometimes used to make verbs which do not refer to a certain person, like "can't be seen" [natavan did], "mustn't be said" [nabayad goft], and "shouldn't be read" [nashayad khand]. Such verbs are called non-personal transitive verbs. (Anvari \& Givi, p.70) The following example makes the point clear:

5).The commandments were written on the tarred wall in great white letters that could be read thirty yards away. (Orwell, 1945, p23)

In this sentence the passive structure "could be read" is translated as "was possible not to be read" [ mishod nakhand]

\section{Personal Transitive Verbs}

Based on the definition of transitive verbs by Anvari \& Givi and also their subcategorization to personal and nonpersonal, all transitive verbs except from the non-personal ones are included in the group of personal transitive verbs. The following is an example:

6).This was sung every Sunday morning after the hoisting of the flag. (Orwell, 1945, p77)

In this sentence the passive structure "was sung" is translated as "they sang it" [mikhandand] in Hosseini \& Nabizade (p.92)

\section{B. Group Two: Passive Structure}

The second group consists of the passive structure. In other words, this group includes the English passive structures which are translated into a passive structure in Persian.

According to Anvri \& Givi (1966), a verb whose subject is not known is a passive one. In other words, the transitive verb can be attributed to the object without mentioning the subject. A passive verb is made by using the past participle of the verb, i.e. the past base form of the verb, plus the final vowel /e/, and different forms of the auxiliary verb "become" [shodan]. (p.50) The following is an example making this point clear:

7).A schoolroom would be built in the farmhouse garden. (Orwell, 1945, p 96)

In this sentence the passive structure "would be built" is translated just like the passive structure in English [sakhte khahad shod]

\section{Group Three: Equivalent Structure}

The third group includes the equivalent structures. For some passive structures, the translators have not used the translation of the same verb in Persian, rather they have used some other verbs whose meanings are similar to the passive verb in English, or they have used some other structures which are identified as noun phrases or idiomatic expressions in this study. So the equivalent structure is divided to three subcategories:

\section{Equivalent Verbs}

This group includes verbs which are not the exact translation of the English passive verb, but rather an equivalent verb having a similar meaning. This group itself is divided to two groups:

\section{Equivalent active verbs}

Here is an example:

8) The very instant that our usefulness has come to an end we are slaughtered with hideous cruelty. (Orwell, 1945, p8) 
In this sentence the passive structure "are slaughtered" is translated as "are destroyed" [az bein miravim] in Firouzbakht's translation (p.9)

\section{Equivalent Passive verbs}

Here is an example:

9).The milk and the windfall apples should be reserved for the pigs alone. (Orwell, 1945, p33)

In this sentence the passive structure "should be reserved" is translated as "should be removed" [kenar gozashte shaved] in Moosaei's translation (p.38)

\section{Noun Phrases}

Some passive structures are translated through another structure named noun phrases. By noun phrases, we mean the nouns or their derived forms which are used to translate English passive structures. According to Anvri \& Givi(1996) a noun is a word which can directly and independently be used as the subject of the sentence and can be the name of a person or something (p.78). They (p.21) have defined infinitive in Persian as the past base form of the verb plus the suffix /an/ which has the meaning of a verb without mentioning the subject or tense, like "to eat" [khordan]. Infinitive is a kind of noun. The following is an example of a noun phrase:

10).When the corn was cut and stacked and some of it was already threshed, a flight of pigeons came whirling through the air. (Orwell, 1945, p36)

In this sentence the passive structures "was cut, stacked and threshed" are translated as "cutting, stacking, and threshing" [deravidan, koppe kardan, and kharman koobi] in Hosseini \& Nabizade's translation (p.43)

11).The sheep who had been killed was given a solemn funeral. (Orwell, 1945, p39)

In this sentence the passive structure "had been killed" is translated as "sacrificing" [ghorbani] in Amirshahi's translation (p.38)

In these examples, there are passive structures which are translated through using noun phrases which can be considered as another capacity to translate English passive structures.

\section{Idiomatic Verbs (Verbal expressions)}

Khanlari (1987, p.169) has defined idiomatic verbs or verbal expressions as a group of words the whole of which conveys a meaning generally equivalent to the meaning of a simple or compound verb. This definition includes all expressions which have the following conditions in addition to the above-mentioned criterion: a) they don't include more than two words, b) one group of the words includes a prepositional phrase, and c) the total meaning is a figurative one, i.e. it doesn't imply the meaning of any of the parts. He gives the example of "being separated from the foot" [az pai dar amadan] which means "to fall down" [oftadan]. The following is an example in the translations which are studied in this research:

12). It was rumoured that a corner of the large pasture was to be fenced off. (Orwell, 1945, p 94)

In this sentence the passive structure "was rumored" is translated as "the rumor had fallen" [cho oftade bood], meaning "it was rumored", in Hosseini \& Nbizade's translation (p.113)

\section{Group Four: Elision}

In this group, there are passive structures which are omitted in the Persian translation. In fact, in such cases the translator has used the surrounding context to present the meaning of the passive structure without using it in the translation of the part. The following is an example:

13) A young pig named Pinkeye was given the task of tasting all his food before he ate it. (Orwell, 1945, p52)

This sentence is translated without the translation of the passive structure "was given the task" in Amirshahi's translation (p.76)

As it is seen in this example, without translating the passive structure, the translator has used some other devices like changing the meaning of the gerund "tasting" to the simple past verb "tasted" [micheshid] to translate the sentence and make the meaning of the original sentence clear.

Based on the different structures used in the translations of the book "Animal Farm" by George Orwell (1945), we can come to the following figure as a summary of all the above-mentioned structures:

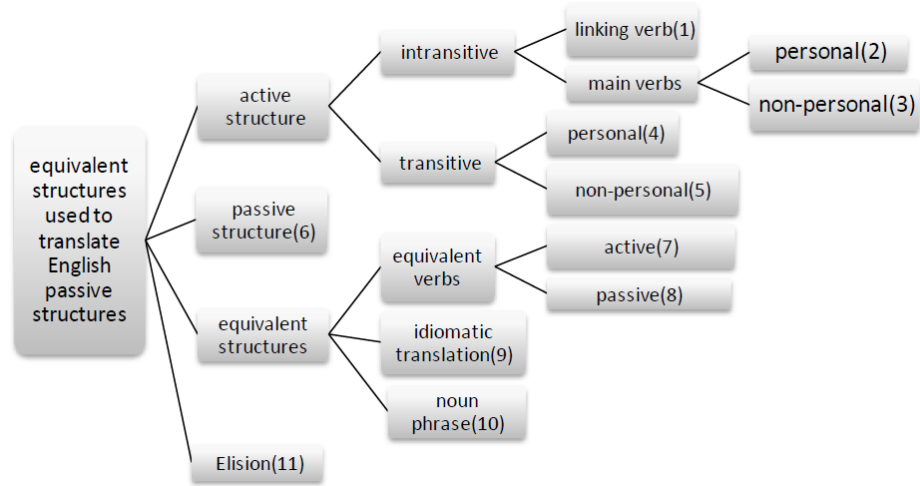

Figure1: Equivalent structures in Persian to translate English passive structures 
The frequency of the above-mentioned eleven structures in each of the five different translations is given in percentages in the following figure:

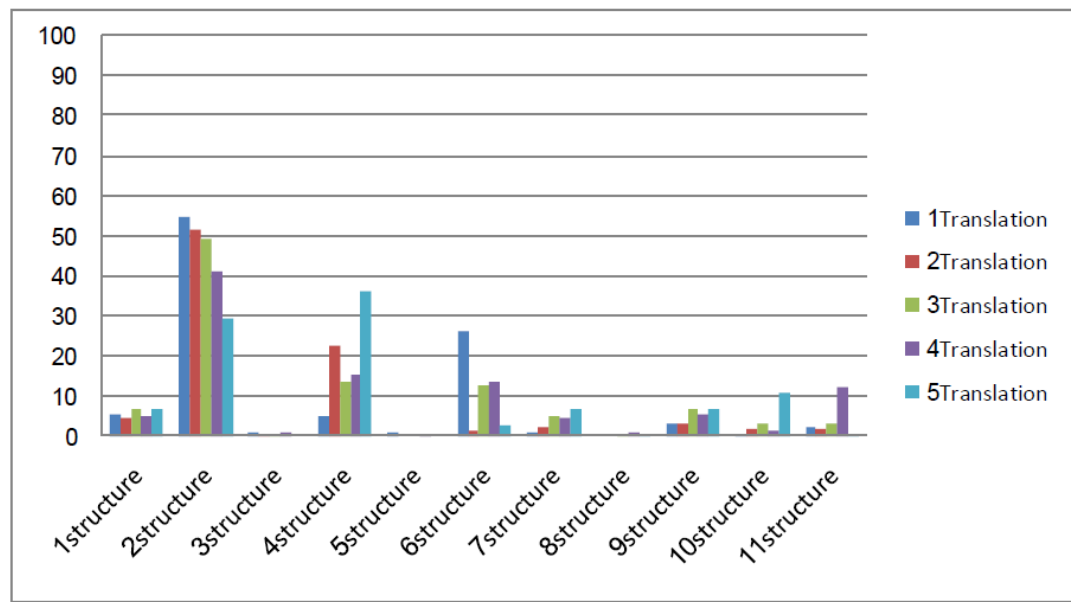

Figure2: Frequency of the eleven structures in each of the five translations Percentage

\section{CONCLUSIONS}

According to the frequency of the eleven structures in the five translations, it can be concluded that the structure "active-intransitive-main verbs (structure2)" is the most frequent one among the structures used to translate the English passive structures, and it is the prevailing tendency among the different translators to use this structure. The frequency of this structure also shows that in Hosseini's translation (No.5), this structure is less frequent than in the other translations. In this translation, the structure "active-transitive-personal (structure4)" is the most frequent one. This structure (No.4) is the second frequent structure among the eleven structures. The average frequency of the two structures 2 and 4 shows that in Persian, the active structure is more common than the passive structure. The passive structure (No.6) places the third among the others. It shows that translators do not believe this structure to be a suitable one to translate English passive structures. The frequencies of structures 1, 7, 9, 10, 11, and 8 fall in the next levels, respectively. Their frequencies are, however, not so different. The least frequent structures are "intransitive-main nonpersonal (No.3)" and "transitive active non-personal (No.5)". As a whole, Persian has various means and structures to translate English passive structures. The frequency of each of these structures is different and seems to depend on the translator's style and his mastery over the two languages. It is also obvious that translators prefer to use the Persian active structure rather than the passive one to translate English passive sentences.

\section{REFERENCES}

[1] Alizade, Z. (2008). Animal Farm, Tabriz: Yaran Publications, $4^{\text {th }}$ Edition.

[2] Anvari, H. \& Ahmadi Givi, H. (1996). Persian Grammar2, Tehran: Fatemi Publications.

[3] Amirshahi,A. (2009). Animal Farm, Tehran: Amir Kabir Publications, $8^{\text {th }}$ Edition.

[4] Bateni, M. (2007). A Description of Persian Grammar, Tehran: Amir Kabir Publications, $19^{\text {th }}$ Edition.

[5] Dabirmoghaddam, M. (1985). "Passive in Persian", Linguistics, Year Two, No.1 pp. 31-46.

[6] Hosseini, S. \& Nabizade,M. (2008). Animal Farm, Tehran: Doostan Publications, $4^{\text {th }}$ Edition.

[7] Firoozbakht, M. (2009). Animal Farm, Tehran: Hekayati Degar Publications, $5^{\text {th }}$ Edition.

[8] Jabbari, M.J. (2003). "The Difference between Passive in Persian and English", Linguistics, Year 18, No.1, pp. 78-94.

[9] Khanlari, P. (1987). The History of the Persian Language, Tehran: Nou Publications, $3^{\text {rd }}$ Edition.

[10] Khanlari, P. (1998). Persian Grammar, Tehran: Toos Publications.

[11] Lambton, A K. S., (1983). Persian Grammar, Cambridge: Cambridge University Press.

[12] Lazar, J. (2005). Contemporary Persian Grammar, Tehran: Hermas Publications.

[13] Lyons, J. (1996). Linguistic Semantics, Cambridge: Cambridge University Press.

[14] Moosaei, H. (2009). Animal Farm, Tehran: Selseleye Mehr Publications, $3^{\text {rd }}$ Edition.

[15] Moyne,J.,(1974), "The so-called passive in Persian", Foundation of Language, 12,249-96.

[16] Orwell, G.,(1983). Animal Farm, U.K: Penguin Books.

[17] Quirk, R. and Greenbaum S. (1973). A concise grammar of contemporary English, New York: Harcourt Brace Jovanovich.

[18] Pakravan, H. (2002). "The especial analysis of passive aspect in Persian Language", Humanities and Social Sciences Magazine, Shiraz University, Year 18, No.1, pp. 36-52.

[19] Shariat, M.J. (1988). Persian Grammar, Tehran: Asatir Publications.

[20] Soheili-Isfahani, A. (1976). Noun Phrase complementation in Persian, Urbana: University of Illinois.

[21] Vahidian Kamyar, T. (2005). "Is there the passive structure in Persian?" Literature Specialized Magazine, Mashhad Azad University, No.7: pp.39-46. 
Omid Tabatabaei is an assistant professor of TEFL at Islamic Azad University, Najafabad Branch. He is currently the Vice-Dean of Humanities Faculty and the Head of English Department. His areas of interest are: L2 acquisition, psycholinguistics, and testing.

Ashraf Rostampour is an MA student of Persian Language and Literature at Islamic Azad University, Najafabad Branch. Her areas of interest are: Persian Literature and Linguistics. 